\title{
Questionário multidimensional para análise da imagem do enfermeiro*
}

\author{
A multidimensional questionnaire to evaluate the image of registered nurses
}

Cuestionario multidimensional para análisis de la imagen del enfermero

\section{Luciana Barizon Luchesi ${ }^{1}$, Isabel Amélia Costa Mendes ${ }^{2}$}

\begin{abstract}
RESUMO
Objetivo: Construir um questionário multidimensional para avaliar a percepção de alunos do ensino médio frente sobre a enfermagem e validar o questionário multidimensional em conteúdo, aparência e análise semântica. Métodos: Estudo quanti-qualitativo com fins de instrumentação, utilizando o referencial teórico-metodológico de Pasquali, Silva e Ribeiro-Filho que recomendam as etapas de levantamento do conceito do constructo, geração dos itens do instrumento fundamentado na literatura e aferição das propriedades psicométricas. Resultados: os itens do instrumento são derivados da literatura sobre psicologia social, história da enfermagem e escolha vocacional. Após validação de conteúdo, validação aparente e análise semântica, houve a aplicação do instrumento em uma amostra de 269 alunos. Conclusão: o instrumento mostrou-se de fácil entendimento e aplicação. Além de análise diagnóstica, o instrumento poderá ser utilizado em estudos experimentais.

Descritores: Papel do profissional de enfermagem; Atitude; Escolha da profissão, Opinião pública; História da enfermagem.
\end{abstract}

\begin{abstract}
Objective: To develop a multidimensional questionnaire to evaluate the perceptions of high school students regarding nursing and to conduct the content, face, and semantic validation of the instrument. Methods: This was a quantitative-qualitative study. The theoretical-methodological approaches of Pasquali, Siva, and Ribeiro-Filho guided the steps of concept/construct definition, development of items, and instrument validation. Results: The items of the instruments were developed from the literature in social psychology, nursing history, and vocational decision. After content, face, and semantic validation, the instrument was pilot tested among 269 students. Conclusions: The items of the instrument were found to be easy to understand. Besides diagnostic analysis, the instrument may be used in experimental studies.
\end{abstract}

Key Words: Role of Nursing Professionals; Attitude; Professional Choice; Public Opinion; Nursing History.

\section{RESUMEN}

Objetivo: Construir un cuestionario multidimensional para evaluar la percepción de los alumnos de la enseñanza media sobre la enfermería y validar el cuestionario multidimensional en contenido, apariencia y análisis semántico. Métodos: Estudio cuantitativo y cualitativo con fines de instrumentación, utilizando el marco teórico metodológico de Pasquali, Silva y Ribeiro-Filho que recomiendan las etapas de levantamiento del concepto del constructo, generación de los ítems del instrumento fundamentado en la literatura y evaluación de las propiedades psicométricas. Resultados: Los ítems del instrumento son derivados de la literatura sobre psicología social, historia de la enfermería y elección vocacional. Después de la validación de contenido, validación aparente y análisis semántico, se aplicó el instrumento en una muestra de 269 alumnos. Conclusión: El instrumento se mostró de fácil entendimiento y aplicación. Además del análisis de diagnóstico, el instrumento podrá ser utilizado en estudios experimentales.

Palabras clave: Papel del profesional de enfermería; Actitud; Elección de la profesión, Opinión pública; Historia de la enfermería.

\footnotetext{
* Extraido de Tese de Doutorado "Análise da imagem do Enfermeiro junto a estudantes do ensino médio: desenvolvimento de Questionário Multidimensional”, apresentada ao Programa de Pós-Graduação em Enfermagem Fundamental da Escola de Enfermagem de Ribeirão Preto da Universidade de São Paulo. Ribeirão Preto (SP), Brasil, (2008), com bolsa da Coordenação de Aperfeiçoamento de Pessoal de Nivel Superior (CAPES).

2 Doutora, Professora do Departamento de Enfermagem Psiquiátrica e Ciências Humanas da Escola de Enfermagem de Ribeirão Preto da Universidade de São Paulo. Ribeirão Preto (SP), Brasil.

${ }_{3}^{3}$ Professora Titular, Departamento de Enfermagem Geral e Especializada da Escola de Enfermagem de Ribeirão Preto da Universidade de São Paulo. Ribeirão Preto (SP), Brasil.
} 


\section{INTRODUÇÃO}

O período de 2006-2015 foi denominado como a década de recursos humanos em saúde, período onde ações centradas na melhoria do acesso à saúde e qualidade da assistência, devem ser o foco do trabalho dos profissionais de saúde ${ }^{(1-3)}$. Dessa forma, o aumento da quantidade de estudantes recrutados para a enfermagem é essencial, visto que vários trabalhos recentes relatam o problema da escassez de profissionais disponíveis no mercado de trabalho mundial ${ }^{4}$ 5). Os países em desenvolvimento devem estar atentos a esse fenômeno, pois a importação de profissionais de países em desenvolvimento para países que já estão em estado de crise de recursos humanos, a longo prazo, provoca sérios problemas na atenção à saúde dos países exportadores de profissionais.

Não existe, ainda, consenso sobre as causas da dificuldade para o recrutamento mundial, mas alguns estudos corroboram a idéia de que a imagem desatualizada que a sociedade possui sobre a enfermagem contribui para essa baixa demanda $a^{(6-7)}$. Por outro lado, a imagem distorcida da profissão, no momento de escolha da carreira, pode favorecer a desistência desse estudante ao longo do curso $^{(8-9)}$.

Através da história da enfermagem, pode-se visualizar as origens de alguns estereótipos e como eles foram enraizados no imaginário social através dos tempos. A maioria deles possui estreita relação com a crença na designação do cuidado como tarefa feminina ao longo da história ${ }^{(10)}$. Nesse sentido, o contexto do estereótipo Enfermagem, enquanto profissão feminina, fez também surgir o preconceito em relação ao homem que opta pela carreira ${ }^{(11)}$. Contudo, a presença masculina na carreira é uma realidade crescente, podendo ser reflexo de rupturas sociais importantes em relação a esse estereótipo ${ }^{(12)}$.

Estereótipos como submissão à medicina, pouco status, representações de caridade ainda são encontrados em estudos recentes sobre o tema em diferentes continentes ${ }^{(6,13-14)}$. Por outro lado, são escassos os estudos sobre a imagem da enfermagem com grupos de estudantes de ensino médio ou estudos que descrevem estratégias educativas para o tema.

A melhoria da imagem social do enfermeiro não tem implicações somente no recrutamento. Em estudo com 80 enfermeiros australianos, foi verificado que estes possuíam imagem mais positiva da enfermagem do que a imagem que eles viam refletida na sociedade. A dissonância causada entre a imagem pessoal (interna) e a percepção da imagem social (externa) que os enfermeiros possuíam da profissão teve implicações sobre a satisfação no trabalho ${ }^{(15)}$.

Considerando-se que o aluno de ensino médio no Brasil é a principal fonte de recrutamento para as profissões, sondagens sobre a imagem que esses estudantes possuem sobre a profissão devem ser realizadas, a fim de verificar se existe, ainda, desatualização da imagem do enfermeiro por essa população e traçar metas de intervenção, se necessário.

Dessa forma, optou-se pela construção de um questionário para que o uso de mais de uma teoria fosse possível, pela escassez de instrumentos brasileiros que abordem esse tema, e a necessidade de utilizar instrumentos de medida validados.

\section{OBJETIVO}

Construir um questionário multidimensional para avaliar a percepção de alunos do ensino médio sobre a enfermagem e validar o questionário multidimensional em conteúdo, aparência e análise semântica.

\section{MÉTODOS}

Trata-se de estudo quanti-qualitativo, tendo como meta a instrumentação que se refere à construção e validação de instrumentos de medida, ou seja, que visa a "construção e validação de instrumento formais, quantitativos, com finalidade de pesquisa ou clínica"(16). Como referencial teóricometodológico para a criação do instrumento foram utilizados os pressupostos de Da Silva e Ribeiro-Filho ${ }^{(17)}$ e Pasquali ${ }^{(18)}$.

De forma geral, os instrumentos de medida devem ser claros, compreensíveis, de fácil aplicação e passíveis de utilização em contextos clínicos diversos. Contudo, deve-se destacar três etapas principais para a construção de um instrumento de medida, recomendados através da literatura e utilizados neste estudo: levantamento do conceito que explica o significado do constructo que se quer medir. Esse passo pode ser alcançado pela revisão de literatura e busca de escalas já construídas. Caso essas escalas já existentes não respondam às necessidades do pesquisador, inicia-se a construção de um instrumento; desenvolvimento do instrumento - geração dos itens do instrumento que sejam relevantes para a mensuração, verificar que tipo de respostas se quer obter e se há necessidade de reduzir itens; aferição das propriedades psicométricas - verificar se o instrumento é válido e fidedigno, por meio de uma série de experimen$\operatorname{tos}^{(17)}$.

Foi solicitada autorização da Diretoria de Ensino - Região de Ribeirão Preto - para realização do estudo. O projeto foi aprovado pelo Comitê de Ética em Pesquisa da Escola de Enfermagem de Ribeirão Preto da Universidade de São Paulo, obtendo aprovação. Durante a coleta de dados os alunos foram esclarecidos sobre os objetivos e procedimentos da pesquisa e que a profissão específica a ser estudada não poderia ser revelada antecipadamente para que os pais não influenciassem a resposta dos estudantes, assim como o pesquisador se apresentou como doutorando da Universidade de São Paulo, para não influenciar as respostas. As justificativas foram explicitadas no Termo de Consentimento Livre e Esclarecido e a pesquisa autorizada pelos órgãos responsáveis. A coleta foi realizada no primeiro semestre de 2007.

\section{RESULTADOS}

Etapas 1 e 2: as escalas internacionais, em sua maioria, apresentam pequenas escalas tipo Likert, abordando os 
estereótipos de forma inadequada para a realidade brasileira (segundo a análise dos pesquisadores do presente trabalho) ou pautando-se apenas na literatura de história da enfermagem, sem utilização de uma teoria da psicologia ou de outra área para a discussão dos resultados e construção das escalas. Nesse sentido, sentiu-se a necessidade de construir um questionário para a realidade brasileira.

Segundo muitos autores na área da psicologia social, o estereótipo refere-se ao componente cognitivo das atitudes. Essas podem ser definidas como "uma organização duradoura de crenças e cognições em geral, dotada de carga afetiva pró ou contra um objeto social definido, que predispõe a uma ação coerente com as cognições e afetos relativos a este objeto" ${ }^{(19)}$. Estereótipos são crenças sobre grupos e indivíduos que podem ser negativos ou positivos e, usualmente, são a base para preconceito e discriminação ${ }^{(20)}$. Desta forma, entender as variáveis que atuam sobre as atitudes e os estere-ótipos pode ajudar na compreensão da formação dos estereótipos relativos à enfermagem, e como intervir no processo.

Entre as variáveis que podem afetar a construção dos estereótipos ou atitudes pode-se citar a família, os meios de comunicação ${ }^{(21)}$, a cultura e a economia, o acesso à informação, interesse pessoal ${ }^{(2)}$, gênero, experiências anteriores $^{(23)}$, classe social, identificação com grupos sociais, autoestima, normas sociais, reforço e punição ${ }^{(19)}$.

Os itens do questionário foram inspirados na literatura de psicologia social, referente aos conceitos de atitude e estereótipos, história da enfermagem, referente ao aparecimento e manutenção de estereótipos, estudos sobre a imagem social do enfermeiro e algumas considerações sobre a escolha da carreira, buscando elementos que poderiam interferir na imagem do profissional entre alunos de ensino médio. $\mathrm{O}$ questionário desenvolvido apresenta mais de uma escala, que, na literatura, é denominado questionário multidimensional, pois tem o "objetivo de medir mais de uma variável e, portanto, possui mais de uma escala"(17).

O questionário foi construído em duas partes: Questio-nário A e Questionário B. No Questionário A são apresen-tadas as questões de identificação, atividades que o estudante realizará ao término do ensino médio, o curso que gostaria de fazer, as características que o atraem para uma profissão, os meios de informação que o estudante utiliza para obter informações sobre a profissão, percepção sobre a influência dos pais na escolha da carreira, status social e financeiro das profissões, profissão dos pais, acesso à internet e aceitação de papéis de gênero para algumas profissões e características. Nesse momento, não fica subentendido o enfoque sobre a enfermagem, a fim de alcançar maior neutralidade nas questões de ranking e aceitação de papéis de gênero para as profissões. Os questionários foram respondidos seqüencialmente.

$\mathrm{O}$ Questionário B verifica o contato que o estudante teve com profissionais da enfermagem, como ele avalia esse contato e a qualidade do atendimento, a diferença entre os profissionais da equipe de enfermagem e o nível de escolaridade, presença de estereótipos, atividades desempenhadas pelo enfermeiro e possibilidade de escolha da enfermagem como carreira.
O Questionário A apresentava, inicialmente, 13 questões, das quais 6 apresentavam sub-itens, não contabilizando, nesse momento, as questões de identificação, data de nascimento e gênero. O Questionário B apresentava, inicialmente, 12 questões, das quais quatro possuíam sub-itens.

Etapa 3: o instrumento foi submetido à validade de conteúdo, validade aparente e análise semântica. A validade de conteúdo enfoca a adequação da amostra da área sobre o conteúdo a ser medido. Especialistas na área dos conteúdos são chamados para analisar a adequação dos elementos na representação do universo hipotético de conteúdos, nas proporções corretas ${ }^{(16)}$. A validade aparente caminha paralelamente à validade de conteúdo, e abrange a compreensão da relevância que os itens parecem ter para os construtores, juízes e indivíduos que compõem a pesquisa ${ }^{(17)}$.

Para alcançar a validade de conteúdo e validade aparente, solicitou-se a avaliação de sete juízes: três psicólogos (dois professores doutores e um especialista), três enfermeiros (doutores, pesquisadores na área de história da enfermagem, análise crítica da assistência de enfermagem ou comunicação em saúde) e um estatístico. Foi solicitado que os avaliadores/ especialistas respondessem se cada item avaliava uma característica ou dimensão do construto pesquisado, em virtude do objetivo apresentado ao lado de cada item. Foi solicitado que os juízes julgassem cada item como: essencial, útil, mas não essencial e não necessário, além de julgar se os itens eram claros e compreensíveis para a população estudada. O item somente foi utilizado quando houve concordância de que o mesmo era essencial para mais de $50 \%$ dos juízes ${ }^{(17)}$.

A validade de conteúdo verifica se a constituição dos itens do instrumento de medida é representativa dentro do conteúdo proposto. Ocorre avaliação mais qualitativa que quantitativa dos itens ${ }^{(17)}$.

Sugestões simples de melhoria de um enunciado, mudança de substantivos por adjetivos, ou inclusão de adjetivos foram feitas para seis questões do Questionário A e oito questões do Questionário B. Uma questão completa e um sub-item foram removidos, pois segundo mais de $50 \%$ dos juízes, apresentava-se repetitivo e um sub-item foi reformulado em uma nova questão (questão 10, Questionário B), por solicitação de mais de $42 \%$ dos juízes.

Um juiz solicitou a inclusão da pedagogia entre as profissões no ranking por se tratar de profissão com conotação social feminina, assim como a enfermagem; portanto, poderia ser um parâmetro de comparação. Houve uma tentativa de responder positivamente a todas as sugestões dos juízes.

Em seguida, elaborou-se a compilação com todas as sugestões e alterações solicitadas e a nova versão foi entregue aos juízes para outra análise, obtendo aprovação unânime, e os itens foram julgados como linguagem compreensível e clara para estudantes de ensino médio.

A análise semântica dos itens permite verificar se são compreensíveis para a população, a fim de evitar deselegância e garantir que a compreensão dos itens não seja fator complicador, ao responder o instrumento. Para realizar a 
análise semântica entre o público-alvo, foi realizado teste piloto em duas escolas de ensino médio escolhidas propositadamente, uma pública (escola Y) e uma privada (escola $\mathrm{X}$ ), representando o extrato mais alto e mais baixo da população ${ }^{(18)}$.

A escola X, de pequeno porte (menos de 100 alunos), encontra-se na região central do município de Ribeirão Preto e a escola $\mathrm{Y}$, de grande porte, encontra-se na região norte. Como o questionário é voltado para alunos do ensino médio, trabalhou-se com uma sala de $1^{\circ}$ ano, uma de $2^{\circ}$ ano e uma de $3^{\circ}$ ano; para isso, foi realizado um sorteio aleatório entre as salas existentes nas escolas.

$\mathrm{Na}$ escola $\mathrm{Y}\left(1^{\circ}\right.$ ano $\left.\mathrm{C}\right)$, dentre os 36 alunos presentes no dia da coleta, dez $(27,8 \%)$ participaram. Foi realizada a leitura do questionário para alunos e solicitada a compreensão sobre cada item e sugestões. Quando o item era composto por características, foi solicitado que os alunos mencionassem sinônimos para as características relacionadas no instrumento. Todas as questões e sugestões foram anotadas pelo pesquisador.

Na escola $\mathrm{X}\left(2^{\circ}\right.$ ano A), dentre os 15 alunos presentes no dia da coleta, $12(80 \%)$ participaram. Foi solicitado que os alunos respondessem o questionário para identificação de possíveis problemas, também que todas as dúvidas fossem expostas para o grupo a fim de identificar se mais alunos apresentavam a mesma dúvida e que os alunos mencionassem a presença de palavras estranhas ao seu vocabulário cotidiano. Na escola X ( $3^{\circ}$ ano A), dentre os 12 alunos presentes no dia da coleta, oito $(66,7 \%)$ participaram. Foi realizado o mesmo procedimento da escola $\mathrm{Y}\left(1^{\circ}\right.$ ano $\left.\mathrm{C}\right)$.

Todas as sugestões dos alunos foram consideradas pertinentes e acatadas e, em sua maioria, referiam-se à facilitação da resposta do aluno, assinalando apenas com um X. As características "passividade", "idealista", "afetuosidade" e "raciocínio crítico" foram removidas, pois não apresentaram fácil entendimento para os alunos. A característica "humanitário" foi substituída por "humano" e "habilidades interpessoais" foi substituída por "habilidade de se comunicar", seguindo a sugestão dos alunos. O item "prestígio social" foi substituído por "profissão que a sociedade valoriza", uma vez que apenas $10 \%$ dos alunos da escola Y compreenderam a característica; contraditoriamente a palavra status não apresentou problemas em nenhuma das escolas. As sugestões foram discutidas com o grupo para verificar a melhoria do entendimento.

Com o propósito de sanar a dificuldade de alguns alunos para o entendimento sobre a possibilidade ou não de repetição de números no escalonamento, para as questões 4, 6 e 7 do Questionário A, e visto que a simples explicação de como as questões deveriam ser respondidas, teve efeito positivo na análise semântica, os autores sugerem que os alunos sejam esclarecidos sobre o preenchimento dessas três questões 10 minutos antes da coleta de dados, tornando assim, o preenchimento mais claro para os alunos.

Considerando que as alterações realizadas no questionário ocorreram mais no sentido de formatação gráfica do que propriamente de entendimento, os pesquisadores consideraram-se satisfeitos com os resultados, nomeando o instrumento como Questionário Multidimensional sobre a Imagem Social do Enfermeiro (QMISE).

A versão final do instrumento foi então aplicada em uma amostra de 269 estudantes de 12 escolas do município de Ribeirão Preto, SP, através de amostragem estratificada das salas de aula existentes em cada escola.

O instrumento mostrou-se de fácil aplicação nas três séries do ensino médio, com tempo de resposta que variou de 25 a 65 minutos. O instrumento mostrou-se muito rico na coleta de informações pertinentes ao estudo da imagem social do enfermeiro. Destaca-se que mais de $70 \%$ da amostra não consideram a possibilidade de escolher a enfermagem como carreira, e não houve diferença estatisticamente significativa entre homens e mulheres para essa resposta.

\section{DISCUSSÃO}

A desatualização da imagem da enfermagem tem sido discutida em nível global. Desta forma, sondagens sobre a visão que estudantes de ensino médio possuem sobre a carreira são importantes para avaliar seu impacto sobre os índices de recrutamento.

A maior parte dos estudos sobre imagem da Enfermagem ainda são descritivos. Apesar do alto valor que estas pesquisas agregam para a discussão do tema, faz-se necessário que os profissionais realizem investimentos em estudos de sondagem e de intervenção, objetivando a melhoria da imagem social do enfermeiro ${ }^{(24)}$. Entretanto, instrumentos estruturados para a realidade de cada cultura devem ser utilizados.

Em muitos casos, é possível adaptar instrumentos de outras culturas, desde que estes satisfaçam as necessidades dos pesquisadores e seja aplicado um rigoroso processo de adaptação transcultural.

Todavia, não foram localizados instrumentos que correspondessem às expectativas dos pesquisadores no presente estudo. Os instrumentos internacionais localizados, em sua maioria, focam a abordagem do instrumento somente nos estereótipos, não possibilitando a correlação com outras variáveis além de gênero.

Desta forma, optou-se pela construção de um questionário multidimensional para a sondagem da imagem do enfermeiro junto a estudantes de ensino médio.

Construir e aferir um instrumento de medida requer um amplo conhecimento do constructo que será mensurado e do processo que deve ser percorrido para testar se este instrumento avalia, discrimina ou prediz este constructo ${ }^{(17)}$.

\section{CONCLUSÃO}

O instrumento de coleta de dados apresentou-se de fácil aplicação e entendimento para os estudantes. Somente algumas palavras necessitaram de substituição ou eliminação, pois não faziam parte do vocabulário dos estudantes. Visto que a imagem social do enfermeiro ainda é um problema presente na sociedade, o QMISE permite não 
somente o estudo da imagem do enfermeiro junto a estudantes do ensino médio, mas pode, também, ser utilizado como estratégia diagnóstica antes de planejar ações de intervenção. Pode-se, ainda, avaliar ações educativas utilizando o QMISE em estudos experimentais. Espera-se que, após intervenções educativas, o índice de estereótipos seja reduzido e o índice de alunos que consideram a possibilidade de escolher a carreira aumente.

A entrada maciça da mulher no mercado de trabalho em profissões antes socialmente classificadas como masculinas abriu um leque maior de oportunidades para a mulher que, antes, optava por maior número de profissões com conotação social feminina.

Os resultados desta pesquisa conduzem a hipótese de que a mudança na configuração do mercado de trabalho já está

\section{REFERÊNCIAS}

1. Pan American Health Organization [Internet]. Toronto: PAHO; 2005. Observatory of Human Resources. [cited 2008 Jan 18]. Available from: http:// www.observatoriorh.org/Toronto/CallAction_ eng1.pdf

2. Mendes IAC, Marziale MHP. Década de recursos humanos em saúde: 2006-2015. Rev Latino-am Enfermagem. 2006;14(1):1-2.

3. Rigoli F, Rocha CF, Foster AA. Desafios críticos dos recursos humanos em saúde: uma visão regional. Rev Latino-am Enfermagem. 2006;14(1):7-16.

4. Grainger P, Bolan C. Perceptions of nursing as a career choice of students in the Baccalaureate nursing program. Nurse Educ Today. 2006;26(1):38-44.

5. Tzeng HM. Testing a conceptual model of the image of nursing in Taiwan. Int J Nurs Stud. 2006;43(6):755-65.

6. Foong AL, Rossiter JC, Chan PT. Socio-cultural perspectives on the image of nursing: the Hong Kong dimension. J Adv Nurs. 1999;29(3):542-8.

7. Luchesi LB, Santos CB. Enfermagem: o que esta profissão significa para adolescentes. Uma primeira abordagem. Rev Latinoam Enferm. 2005;13(2):158-64.

8. Brodie DA, Andrews GJ, Andrews JP, Thomas GB, Wong J, Rixon L. Perceptions of nursing: confirmation, change and the student experience. Int J Nurs Stud. 2004;41(7):721-33.

9. Vieira MJ. A representação do cuidar na imagem cultural da enfermagem. Rev Latino-am Enfermagem. 1999; $7(5): 25-31$.

10. Nauderer TM, Lima MADS. Imagem da enfermeira: revisão da literatura. Rev Bras Enferm. 2005;58(1):74-7.

11. Evans, JA. Cautious caregivers: gender stereotypes and the sexualization of men nurses' touch. J Adv Nurs. 2002;40(4): 441-8. exercendo influência nos estudantes de ensino médio. Caso essa hipótese seja confirmada em estudo futuro, aumenta a preocupação para a enfermagem, pois denota dificuldade de recrutamento, também para o gênero feminino.

É primordial que ações diretivas sejam estabelecidas, a fim de que a Enfermagem tenha sua imagem social atualizada, e o profissional sinta-se reconhecido pelo valoroso trabalho que presta à sociedade. Entretanto, é necessário que o fenômeno continue a ser estudado e que ações educativas sejam avaliadas e incentivadas.

\section{Agradecimento}

Aos juízes do questionário por sua valorosa contribuição ao trabalho, às escolas e aos alunos participantes.

12. Coelho EAC. Gênero, saúde e enfermagem. Rev Bras Enferm. 2005;58(3):345-8.

13. Kemmer LF, Silva MJP. Como escolher o que não se conhece? Um estudo da imagem do enfermeiro por alunos do ensino médio. Acta Paul Enferm. 2007;20(2):125-30.

14. Rossiter JC, Yam B. Promoting the nursing profession: the perceptions of non-English-speaking background high school students in Sydney, Australia. Int J Nurs Pract. 1998;4(4):213-9.

15. Takase M, Kershaw E, Burt L. Nurse-environment misfit and nursing practice. J Adv Nurs. 2001;35(6):819-26.

16. Polit DF, Beck CT, Hungler BP. Fundamentos de pesquisa em enfermagem: métodos, avaliação e utilização. 5a ed. Porto Alegre: Artmed; 2004.

17. Da Silva JA, Ribeiro-Filho NP. Avaliação e mensuração de dor: pesquisa, teoria e prática. Ribeirão Preto: FUNPEC Editora; 2006.

18. Pasquali L. Psicometria: teoria dos testes na psicologia e na educação. Petrópolis: Vozes; 2003.

19. Rodrigues A, Assmar EML, Jablonski B. Psicologia social. 22a ed. Petrópolis: Vozes; 2003.

20. Breckler SJ, Olson JM, Wiggins EC. Social psychology alive. Belmont, CA: Thomson/ Wadsworth; 2006.

21. Pisani EM, Pereira S, Rizzon LA. Temas de psicologia social. Petrópolis: Vozes; 1994.

22. Asch SE. Psicologia social. 3a ed. São Paulo: Nacional; 1972.

23. Baron RA, Byrne D. Social psychology. 8th ed. Boston: Allyn and Bacon; c1997.

24. Droes NS, Hatton DC, Kramer M. Effects of a participative intervention on high school students' image and valuation of nursing. J Prof Nurs. 1993;9(1):41-9. 
Questionário Multidimensional para Imagem Social do Enfermeiro (QMISE)

QUESTIONÁRIO A

Data Nascimento ___

\begin{tabular}{|l|lrl|}
\hline 1. Ao terminar o colegial o que você vai fazer em relação aos estudos? \\
\hline 1-Parar de estudar $\square$ & 2-Cursinho $\square \quad$ 3-Curso técnico $\square$ & 4-Faculdade $\square$ \\
\hline 1-Vai trabalhar $\square$ & 2. Ao término do colegial você... \\
\hline
\end{tabular}

\section{Se você tivesse oportunidade de cursar uma faculdade qual curso você gostaria de fazer?}

\begin{tabular}{|c|c|c|}
\hline \multicolumn{3}{|c|}{$\begin{array}{l}\text { 4. Enumere de } 1 \text { a } 10 \text {, sendo } 1 \text { o fator MAIS IMPORTANTE até } 10 \text { o MENOS IMPORTANTE. } \\
\text { Quais os aspectos mais importantes que uma profissão deve possuir para que você a escolha? }\end{array}$} \\
\hline $\mathrm{N}^{0}$ & Fatores & \begin{tabular}{l|l}
$\mathbf{N}^{\circ}$ & Fatores \\
\end{tabular} \\
\hline & Possibilidade de conciliar trabalho e familia & Profissão que a família apóia \\
\hline & Trabalho que não causa estresse & Realização pessoal \\
\hline & Profissão valorizada pela sociedade & Salários altos \\
\hline & Poucas horas de trabalho semanais & Não trabalhar de fim de semana, ou à noite \\
\hline & Possibilidade de ser chefe & Profissão com muita oferta de emprego \\
\hline
\end{tabular}

a)Além dos fatores acima citados, existe algum fator que você considera importante? Qual

\section{Para as questões abaixo assinale sua resposta com $X$.}

a) Você sabe quais as faculdades ou escolas que tem o curso que você escolheu?

b) Você sabe quanto ganha em média o profissional?

c) Você já conversou com um profissional para esclarecer dúvidas?

d) Você buscou informações em jornais, revistas, internet?

e) Você precisa sair de Ribeirão para fazer o curso que você escolheu?

f) Você tem apoio da família para a profissão que você escolheu?

6. Enumere de 1 (a que tem mais status social) até 15 (a que tem menos status social). Das profissões abaixo quais são, em sua opinião, as que têm mais destaque (status, prestígio) na sociedade?

\begin{tabular}{|l|l|}
\hline Número & Profissão \\
\hline & Fonoaudiologia \\
\hline & Direito \\
\hline & Administração \\
\hline & Engenharia \\
\hline & Psicologia \\
\hline
\end{tabular}

\begin{tabular}{|l|l|}
\hline Número & Profissão \\
\hline & Letras \\
\hline & Fisioterapia \\
\hline & Odontologia \\
\hline & Serviço Social \\
\hline & Farmácia \\
\hline
\end{tabular}

\begin{tabular}{|l|l|}
\hline Número & Profissão \\
\hline & Medicina \\
\hline & Enfermagem \\
\hline & Nutrição \\
\hline & Computação \\
\hline & Pedagogia \\
\hline
\end{tabular}

a)Existe alguma outra profissão além destas que você destacaria? Qual ou quais?

7. Dentre as profissões listadas abaixo, quais são, em sua opinião, as que oferecem salários mais altos. Enumere de 1 (a que ganha mais dinheiro) até 15 (a que ganha menos dinheiro).

\begin{tabular}{|l|l|}
\hline Número & Profissão \\
\hline & Fonoaudiologia \\
\hline & Direito \\
\hline & Administração \\
\hline & Engenharia \\
\hline & Psicologia \\
\hline
\end{tabular}

\begin{tabular}{|l|l|}
\hline Número & Profissão \\
\hline & Letras \\
\hline & Fisioterapia \\
\hline & Odontologia \\
\hline & Serviço Social \\
\hline & Farmácia \\
\hline
\end{tabular}

\begin{tabular}{|l|l|}
\hline Número & Profissão \\
\hline & Medicina \\
\hline & Enfermagem \\
\hline & Nutrição \\
\hline & Computação \\
\hline & Pedagogia \\
\hline
\end{tabular}

a)Além destas, existe alguma outra profissão que, em sua opinião, oferece salários altos? Qual ou quais? 


\section{Qual é a profissão de seus pais?}

Mãe

Pai

9. Qual o grau de escolaridade dos seus pais?

\section{Mãe}

0 - Analfabeto

1 - $1^{\circ}$ Grau incompleto (não chegou até a $8^{a}$. Série)

2 - $1^{\circ} \mathrm{Grau}$ completo (terminou a $8^{\mathrm{a}}$. série)

3 - $2^{\circ} \mathrm{Grau}$ incompleto (não chegou até o $3^{\circ}$ colegial)

4 - $2^{\circ}$ Grau completo (terminou o $3^{\circ}$ colegial)

5 - $3^{\circ} \mathrm{Grau}$ incompleto (não terminou a faculdade)

6. $-3^{\circ} \mathrm{Grau}$ completo (terminou a faculdade)

\section{Pai $\square$}

0 - Analfabeto

1 - $1^{\circ} \mathrm{Grau}$ incompleto (não chegou até a $8^{\mathrm{a}}$. Série)

2 - $1^{\circ} \mathrm{Grau}$ completo (terminou a $8^{\mathrm{a}}$. série)

3 - $2^{\circ} \mathrm{Grau}$ incompleto (não chegou até o $3^{\circ}$ colegial)

4 - $2^{\circ}$ Grau completo (terminou o $3^{\circ}$ colegial)

5 - $3^{\circ} \mathrm{Grau}$ incompleto (não terminou a faculdade)

6. - $3^{\circ}$ Grau completo (terminou a faculdade)

\section{Para as questões abaixo assinale sua resposta com $X$.}

a. Seus pais dão opiniões ou conselhos sobre o que você vai fazer depois do colegial (trabalhar, faculdade, curso, etc)?

b. A vida profissional dos seus pais influenciou a sua escolha de carreira?

c. Você tem o hábito de ler jornal?

d.Você tem o hábito de ver noticiário na televisão?

e.Você tem o hábito de freqüentar palestras, seminários ?

f.Você tem o hábito de freqüentar biblioteca?

\begin{tabular}{|c|c|c|c|}
\hline \multicolumn{4}{|c|}{ 11. Você usa a internet ?: } \\
\hline 1-Muito & 2- Razoavelmente & 3- Pouco & 4 -Não uso \\
\hline
\end{tabular}

\section{Para as questões abaixo responda:}

1 - Profissão melhor desempenhada por HOMENS

2 - Profissão melhor desempenhada por MULHERES

3 - Indiferente, melhor desempenhada por AMBOS

4 - Não sei

\begin{tabular}{|l|l|}
\hline Número & Profissão \\
\hline & Computação \\
\hline & Medicina \\
\hline & Odontologia \\
\hline & Psicologia \\
\hline & Direito \\
\hline
\end{tabular}

\begin{tabular}{|l|l|}
\hline Número & Profissão \\
\hline & Nutrição \\
\hline & Farmácia \\
\hline & Fisioterapia \\
\hline & Engenharia \\
\hline & Fonoaudiologia \\
\hline
\end{tabular}

\begin{tabular}{|l|l|}
\hline Número & Profissão \\
\hline & Enfermagem \\
\hline & Serviço Social \\
\hline & Letras \\
\hline & Administração \\
\hline & Pedagogia \\
\hline
\end{tabular}

\begin{tabular}{|c|c|c|c|}
\hline \multicolumn{4}{|c|}{ 13. Para as questões abaixo responda se as características são mais : } \\
\hline 1- MASCULINAS & 2- FEMININAS & 3- DE AMBOS & 4- Não Sei \\
\hline Sensibilidade & & Frieza & Timidez \\
\hline Maturidade & & Ousadia & Responsabilidade \\
\hline Criatividade & & Independência & Solidariedade \\
\hline Agressividade & & Liderança & Competitividade \\
\hline Comprometimento & & Compreensão & Delicadeza \\
\hline Honestidade & & Paciência & \\
\hline Competência & & Realismo & \\
\hline
\end{tabular}

